\title{
L'énoncé normatif et le lexique juridique
}

\author{
Joaquín Giráldez Ceballos-Escalera \\ Universidad Nacional de Educación a Distancia (UNED) \\ jgiraldez@flog.uned.es
}

\section{L'énoncé normatif}

«L'énoncé normatif est celui qui formule un ordre, en permettant, obligeant ou interdisant une conduite. Il s'oppose à l'énoncé qui reconnaît, constate ou décrit une situation, promeut, favorise, encourage, ou encore exprime des vœux, des souhaits, des recommandations ou des avis » (Champeil-Desplats, 2006 : 4).

« L'énoncé législatif est conçu de manière à faire reconnaître la souveraineté de celui dont il émane, et le caractère obligatoire de ce qu'il édicte » (Cornu, $1990: 267$ ).

\section{Absence de portée normative}

Le 3 janvier 2005, à l'occasion des ses vœux au président de la République, le président du Conseil constitutionnel, Pierre Mazeaud, a lancé une sévère mise en garde contre la « mauvaise santé législative » en manifestant que « la loi n'est pas faite pour affirmer des évidences, émettre des vœux ou dessiner l'état idéal du monde (en espérant sans doute le transformer par la seule grâce du verbe législatif ?). La loi ne doit pas être un rite incantatoire. Elle est faite pour fixer des obligations et ouvrir des droits. » (Les Cahiers du Conseil Constitutionnel 18 / 2005 : 10).

Le président du Conseil constitutionnel dénonçait la multiplication dans la loi des déclarations d'intention juridiquement vides : «j'ai fait procéder à un recensement exhaustif de la centaine d'occurrences du mot « loi » figurant dans nos textes de rang constitutionnel.

Ce recensement fait apparaître que la loi est le sujet de verbes ayant tous un contenu « décisoire » (détermine, fixe, ordonne, régit, réglemente, autorise, défend, exclut, etc.).

Le Conseil constitutionnel est donc prêt à censurer désormais les «neutrons législatifs » (Les Cahiers du Conseil Constitutionnel 18 / $2005: 12$ ).

Le « neutron législatif », expression employée par Jean Foyer ${ }^{1}$, aussi appelé droit souple, droit mou, etc. est une loi sans contenu obligatoire, qui ne va avoir aucun effet en pratique.

La liste de ces « neutrons législatifs » qui « encombrent la législation » est longue :

Le 21 avril 2005, le Conseil constitutionnel a déclaré pour la première fois une disposition législative contraire à la Constitution pour absence manifeste de portée normative: l'article 12 de la Loi «d'orientation et de programme pour l'avenir de l'école», dite Loi Fillon « fait référence à un texte de portée politique mélangeant les souhaits, les pétitions de principe et des objectifs [...]. Cet article dépourvu de portée normative ne peut qu'encourir la censure ${ }^{2} \gg$.

La Loi Fillon n'est pas un cas unique, le défaut de normativité de plusieurs dispositions législatives est fréquent $^{3}$ :

Il est possible de recenser des lois ...

qui expriment des souhaits :

Art. $1^{\text {er }}$ de la loi nº 68-978 du 12 novembre 1968 d'orientation de l'enseignement supérieur 
« Les universités doivent s'attacher à porter au plus haut niveau et au meilleur rythme de progrès les formes supérieures de la culture».

qui constatent une situation :

\section{- Art. $1^{\text {er }}$ de la loi no ${ }^{94-629}$ du 25 juillet 1994 relative à la famille}

«La famille est une des valeurs essentielles sur lesquelles est fondée la société. C'est sur elle que repose l'avenir de la nation. A ce titre, la politique familiale doit être globale».

qui décrivent un fait :

- Art. $1^{\text {er }}$ de la loi $n^{\circ}$ 77-2 du 3 janvier 1977 sur l'architecture

«L'architecture est une expression de la culture».

qui expriment des vœux :

- Art. $1^{\text {er }}$ de la loi $n^{\circ} 84-610$ du 16 juillet 1984 relative à l'organisation et à la promotion des activités physiques et sportives

"Les activités physiques et sportives constituent un facteur important d'équilibre, de santé, d'épanouissement de chacun; elles sont un élément fondamental de l'éducation, de la culture et de la vie sociale. Leur développement est d'intérêt général et leur pratique constitue un droit pour chacun quels que soient son sexe, son âge, ses capacités ou sa condition sociale ».

qui reconnaissent un fait :

- Art. $1^{\text {er }}$ de la loi no $2001-70$ du 29 janvier 2001 relative au génocide arménien de 1915

« La France reconnaît publiquement le génocide arménien de 1915 ».

On assiste à la transformation du discours normatif en un discours qui, au lieu d'interdire ou d'obliger, formule des recommandations ; au lieu de déterminer, fixer, établir ou ordonner une conduite, suggère une direction.

Ce changement sémantique a aussi transformé les verbes défendre, interdire, devoir, obliger, falloir, etc. par des expressions s'appuyant sur la responsabilité ou la flexibilité. Mais ces énoncés sans valeur normative, ni force contraignante, ne constituent pas de vraies règles de droit, puisque une règle de droit doit être générale, obligatoire et sanctionnée.

Ces lois appelées lois d'orientation ou lois de programmation marquent une intention politique ou une déclaration de principe dont la portée a été précisée par la jurisprudence du Conseil constitutionnel qui s'est refusé à censurer les dispositions législatives sans portée normative, jugeant que, ne pouvant pas produire d'effets juridiques, elles ne peuvent pas être déclarées inconstitutionnelles, parce qu'elles figurent à l'article 34 de la Constitution qui dispose que « Des lois de programmation déterminent les objectifs de l'action de l'Etat ».

Celles-ci ne devraient figurer que dans l'exposé des motifs de la loi et non dans son dispositif.

\section{L'énoncé normatif et la logique déontique}

Selon la logique déontique, l'énoncé normatif formalise les rapports qui existent entre les quatre alternatives d'une loi : l'obligation, l'interdiction, la permission et le facultatif. La loi a pour vocation d'énoncer des règles et doit par suite être revêtue d'une portée normative.

Le caractère normatif est introduit par les auxiliaires déontiques devoir, pouvoir, obliger, falloir, puisque, « la forme grammaticale seule ne permet pas de distinguer un énoncé propositionnel normatif (déontique) d'un énoncé propositionnel non normatif (non déontique), d'une proposition théorique indicative par exemple ou d'une proposition pratique impérative » (Kalinowski, 1972:19). 


\section{Lexique et énoncé normatif}

Gémar (1991 : 276) affirme que le langage de spécialité est composé d'un «noyau de termes qui associés à des cooccurrents précis et à des mots de la langue générale réaliseraient le texte d'un discours spécialisé donnée, dont la somme apporterait au texte la plénitude de sa signification ». Cet auteur explique l'existence du langage juridique à partir de la formule suivante :

\section{$\frac{\text { TERME }}{\text { COOCCURRENT(s) }+ \text { Vogén }}=$ LS}

L'énoncé normatif est composé de trois éléments : terme, cooccurrent et vocabulaire général :

Terme : délit

Cooccurrent : commettre

Vogén: quiconque, un, sera puni, sévèrement

«Quiconque commet un délit sera puni sévèrement »

Sur le plan morphosyntaxique, l'énoncé normatif est constitué par un opérateur déontique se caractérisant par :

(i) l'emploi des verbes exprimant

- l'obligation : devoir, obliger, falloir...

«Le juge doit se prononcer sur tout ce qui est demandé et seulement sur ce qui est demandé ». (N.C.P.C. art. 5)

- l'interdiction : défendre, interdire, ne pas pouvoir...

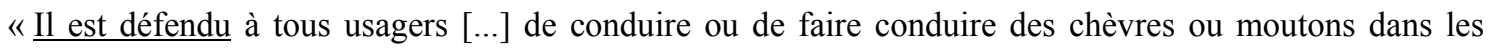
forêts et sur les terrains qui en dépendent $[. .$.$] » (C. forestier, art. L138-10.)$

- la sanction : punir, déchoir, condamner...

« Celui qui chasse sur un terrain où il n'a pas le droit de chasser sera puni d'une amende de $25.000 \mathrm{~F}$ au plus ou de l'emprisonnement pendant trois mois au plus » (C. rural, art. L229-31.)

- les facultés ou la reconnaissance des droits : permettre, avoir droit, pouvoir...

«Il est permis aux propriétaires d'établir sur leurs propres propriétés, ou en faveur de leurs propriétés, telles servitudes que bon leur semble [...]» (C. civ. art. 686).

(ii) l'expression de la généralité et l'abstraction de la règle de droit :

La règle de droit est impersonnelle et générale ${ }^{4}$. Elle s'applique à tous les individus. Pour l'exprimer, l'énoncé normatif utilise des pronoms et des adjectifs indéfinis :

« Toute servitude est éteinte lorsque le fonds à qui elle est due, et celui qui la doit, sont réunis dans la même main » (C. Civ. art. 705)

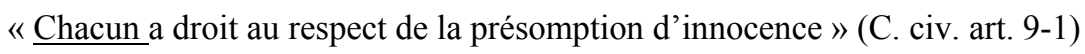

« Nul ne peut être adopté par plusieurs personnes, si ce n'est par deux époux » (C. civ. art. 346)

« Celui qui réclame l'exécution d'une obligation doit la prouver » (C.civ. art. 1315)

(iii) l'utilisation des formules performatives verbales :

Les décisions normatives et notamment les décisions judiciaires (jugements, arrêts) utilisent les verbes performatifs dont l'énonciation constitue un acte en soi : «parler c'est agir» (Austin : 1970).

Lorsque le juge prononce le divorce, il dissout du même fait le mariage. «Le prononcé du divorce a en lui-même une efficacité juridique » (Garapon, 1997 : 139). 
« Je prononce votre divorce et $\mathrm{j}$ 'homologue votre convention ».

PAR CES MOTIFS :

Le tribunal

Statuant publiquement, par jugement réputé contradictoire et en premier ressort ;

PRONONCE la nullité du contrat intitulé « demande d'adhésion » conclu le 15 juillet 1999 ;

CONDAMNE solidairement les sociétés ... à restituer aux époux XXXXXX la somme de XXXX

DEBOUTE les époux XXXXXX de leur demande de dommages-intérêts ;

DIT n'y avoir lieu à exécution provisoire ;

TGI Angoulème, lère Chambre, 14 déc. 2000

Le Tribunal,

Statuant publiquement, contradictoirement et en premier ressort,

Vidant son délibéré du 22janvier 2003,

Déboute la société ZD NET FRANCE S.A.S. de ses exceptions d'incompétence et d'irrecevabilité;

Reçoit M. FREDERIC M. en sa demande mais le DIT mal fondé,

En conséquence le déboute;

Dit que les diverses demandes de mise hors de cause et d'appel en garantie sont sans objet;

Déboute les sociétés “ZIFF DAVIS FRANCE”, "ZD NET FRANCE S.A.S.", "VNU PUBLICATIONS FRANCE S.A.”, “SPRAY NETWORK S. A.”, “WSTORE S.A.R.L.”, “KELKOO S.A.” anciennement dénommée KELKOO.COM et “LE NOUVEL OBSERVATEUR DU MONDE” de leurs demande de dommages-intérêts pour procédure abusive; Dit n'y avoir lieu à application de l'article 700 du Nouveau Code de Procédure Civile;

Condamne M. Frédéric MILLOT aux entiers dépens qui seront recouvrés conformément aux dispositions de l'article 699 du Nouveau Code de Procédure Civile;

(iv) les constructions passives inachevées sans complément d'agent (Sourioux et Lerat, 1975 :45) :

La diathèse passive sans complément d'agent renforce l'objectivité de la norme et sa dépersonnalisation (Charrow, Crandall \& Charrow, $1982: 176)$ :

« [...] la nullité ne sera pas prononcée si sa cause a disparu au moment où le juge statue » (code de procédure civile 121)

« Le testament sera ouvert s'il est cacheté » (Code civil 1007)

« La rente viagère ne peut être stipulée insaisissable que lorsqu'elle a été constituée à titre gratuit » (code civil 1981)

« [...] le jugement a été rendu par défaut » (code de procédure civile 540)

« l'opposition est faite selon le mode prévu à l'article 573 »(code de procédure civile 575)

« une condamnation a été prononcée au profit ou à l'encontre d'une personne qui n'était pas partie à l'instance » (code de procédure civile 611)

(v) l'emploi du présent de l'indicatif ayant valeur impérative :

«La lettre de notification informe le salarié qu'il dispose d'un mois à compter de sa réception pour faire connaître son refus » (C. du travail Art. L1222-6) [doit informer le salarié]

(vi) l'usage du verbe devoir + infinitif passif :

« Doit être condamné au versement de dommages-intérêts le mari » (Cour de Cassation, Chambre civile 2, NO 91-17.680 20/01/1993) 
« Doit être cassé pour violation de la loi l'arrêt qui confie à un tiers l'enfant naturel » (Cour de Cassation, Chambre civile 1, NO 91-20.657 13/04/1992)

«le paiement doit être fait au domicile du débiteur » (code civil 1247)

(vii) l'emploi d'une préposition + infinitif passif hérité du moyen âge

«les enfants mineurs ont un droit absolu à être entretenus par leurs parents» (Cour d'appel, Caen 08/06/1989)

«Cette désignation n'a pas à être certifiée » (code civil 2149)

«La pension alimentaire cesse de plein droit d'être due si le conjoint qui en est créancier contracte un nouveau mariage » (code civil 283)

«L'action est le droit, pour l'auteur d'une prétention, d'être entendu sur le fond de celle-ci afin que le juge la dise bien ou mal fondée » (code de procédure civile 30)

(viii) l'utilisation des formules

Une particularité du discours juridique est constituée par les formules, constructions figées possédant une connotation archaïque, maintenues tout au long des temps avec la finalité de donner une solennité aux actes juridiques et, grâce à son rythme, faciliter sa mémorisation.

Dans la formule du serment « Jurez de dire toute la vérité, rien que la vérité », le caractère emphatique est donné par la répétition du mot vérité, ce qui met en évidence l'héritage hébraïque de certaines formules ; en hébreu le superlatif consistait à répéter trois fois le mot (Garapon, 1997 : 138).

L'usage des «doublets» ou «binomials» (Malkiel, 1959) est fréquent dans le discours juridique. Les doublets ou « legal pairs » constituent des tournures composées de deux ou plusieurs termes synonymes, contigus ou complémentaires:

«Je jure de bien et fidèlement remplir mes fonctions ...»

« Jurez et promettez ....

«Parlez sans haine et sans crainte ...»

Ce « rythme binaire » ou « binomial » représente une tautologie, dans le sens qu'il répète de forme inutile la même idée sous une autre forme (Houbert, $2004: 3$ ).

L'arrêt ci-dessus casse et annule la décision en toutes ses dispositions.

Lorsque la demande en divorce est mal fondée [...] le juge la rejette et refuse en conséquence d'homologuer la convention.

« Je jure de bien et fidèlement remplir mes fonctions ...»

«l'arrêt qui statue au fond par la même décision que celle qui écarte le déclinatoire de compétence doit être déclaré nul et non avenu »

Pour certains auteurs, l'origine de cette tautologie est l'habitude des juristes anglais du Moyen-Âge d'associer un terme d'origine anglo-saxonne à un terme latin ou français, à la prudence de certains juristes ou à une fonction esthétique (Hubert, $2004: 4$ ).

L'usage des hyponymes à travers des listes détaillées, dont le signifié est hiérarchiquement plus spécifique que celui d'un autre. Tandis que les synonymes sont mutuellement substituables, le terme générique peut se substituer à l'hyponyme, mais non l'inverse (Martinet, $1969: 193)$ :

«Quiconque aura usurpé l'un des titres d'ingénieur agronome, d'ingénieur agricole, d'ingénieur des industries agricoles et alimentaires ou d'ingénieur horticole sera puni des peines prévues par l'article $259 \mathrm{du}$ code pénal » (code rural L815-3)

Parfois ces hyponymes font référence à des actions : 
"Quiconque aliène, loue ou concède un territoire classé en réserve naturelle est tenu de faire connaitre à l'acquéreur, locataire ou concessionnaire, l'existence du classement » (code rural L242-7)

L'usage d'une anaphore archaïque ou inusuelle, consistant à rappeler un mot ou groupe de mots précédemment énoncé par un terme grammatical :

«En cas que le donateur se soit réservé la liberté de disposer d'un effet [...], ledit effet ou ladite somme appartiendra aux héritiers du donateur [...]» (code civil 946)

« [...] Il y a lieu pour la cour d'inviter l'intéressé à régulariser sa défense en la faisant présenter par l'un des mandataires désignés audit article » (cour administrative d'appel, Paris, chambre 2, NO 90PA00637 03/03/1992)

L'usage de la redondance d'éléments dans un texte permettant de mieux le comprendre constitue une caractéristique prosodique du discours juridique notamment le discours judiciaire («attendu que », « considérant que », « vu que »).

Cette récurrence de mêmes structures énonciatives est définie par Greimas comme un « ensemble redondant de catégories sémantiques qui rend possible la lecture uniforme du récit, telle qu'elle résulte des lectures partielles des énoncés et de la résolution de leurs ambiguités qui est guidée par la recherche de la lecture unique » (Greimas : 1966).

Attendu que la Banque Pouyanne et M. David P. expliquent avoir reçu [...]

Attendu que les demandeurs indiquent avoir $[\ldots]$

Attendu qu'ils critiquent cette information [...]

Attendu qu'ils indiquent et soutiennent que $[\ldots]$

Attendu qu'ils précisent que ce manquement est une faute [...]

Attendu que par conclusions la société Optima on line soutient in limine litis que [...]

Attendu que très subsidiairement la défenderesse soutient qu'il n'est pas démontré [...]

Attendu qu'elle sollicite $5000 €$ sur le fondement de l'article $700 \mathrm{du}$ NCPC.

TGI de Bayonne Ordonnance de référé du 12 juin 2002

Au contraire, pour éviter les répétitions, quand on fait mention des articles visés dans un arrêt ou un texte législatif, on a recours à des structures syntaxiquement différentes, mais partageant le même sens propositionnel :

- au regard de l'article 1382 du code civil

- au sens de la loi du 5 juillet 1985

- au terme de l'article 47 du nouveau code de procédure civile

- conformément à l'article R. 433-1 du code de la construction et de l'habitation

- dans les conditions définies à l'article 6 du décret du 30 septembre 1953

- dès lors les dispositions de l'article R. 516-16 du code du travail

- en application de l'article 1034 du code de procédure civile

- en conformité avec les dispositions du décret du 30 décembre 1964.

- en vertu de l'article 468 du nouveau code de procédure civile.

- fondé sur l'article 28 du décret du 30 septembre 1953

- Il résulte de la loi du 1624 août 1790 et de l'article L. 199 du livre des procédures fiscales

- l'article 85 du nouveau code de procédure civile prévoit... 
- par application de l'article 631 du code de commerce

- selon l'article 31 point 1 de la convention de Vienne du 18 avril 1961

- $\quad$ sur le fondement de l'article 145 du nouveau code de procédure civile.

- visé par l'article 92 du traité de Rome

L’usage de séquences multinominales énumératives de termes contigus pour la précision et l'exhaustivité :

« Les dispositions testamentaires sont ou universelles, ou à titre universel, ou à titre particulier » (code civil. Art. 1002)

« Hors ces deux cas, le juge doit ou adjuger ou rejeter purement et simplement la demande » (code civil Art 1367)

«A défaut d'aucun ascendant, le frère ou la soeur, l'oncle ou la tante, le cousin ou la cousine germains, majeurs, ne peuvent former aucune opposition que dans les deux cas suivants[...]» (code civil Art. 174)

«La possession est la détention ou la jouissance d'une chose ou d'un droit que nous tenons ou que nous exerçons par nous-mêmes ou par un autre qui la tient et qui l'exerce en notre nom [...] » (cour de Cassation, Chambre civile 3, NO 87-18.747 24/01/1990)

L'utilisation de l'hypothèse :

« Toute règle est en soi la réponse à une question » (Cornu, $1990: 283$ )

La norme détermine une hypothèse dont la validité de l'acte juridique est subordonnée à la réalisation d'un événement.

C'est une proposition subordonnée conditionnelle exprimant dans quel cas ou à quelle condition a lieu ou aurait lieu ce qui est énoncé par la proposition principale.

La proposition conditionnelle est constituée d'une protase, placée avant la proposition principale appelée apodose et introduite par une conjonction de subordination ou une locution conjonctive de subordination telle que « en cas », « dans le cas », « si », « quand », « lorsque », etc. Chaque proposition conditionnelle repose sur une formule de base : la protase exprime la condition suffisante, dont l'apodose exprime la conséquence.

«En cas de mort naturelle ou civile de la personne qui a fait le dépôt, la chose déposée ne peut être rendue qu'à son héritier » (C. civ. art.1939)

«Si la décision émane du premier président de la cour d'appel, elle peut être modifiée dans les mêmes conditions par celui-ci » (NCPC. art. 724)

Habituellement, la loi détermine les conséquences juridiques : une obligation, affirmation d'un droit, sanction, etc.

Hypothèse / condition = conséquence juridique

« Si le coupable du délit défini à l'article L. 229-31 se livre professionnellement à la chasse prohibée, il sera puni d'un emprisonnement de trois mois au moins » (code rural L229-33)

Dans la plupart des cas, la protase est placée avant l'apodose :

«Quand le majeur en curatelle demande une autorisation supplétive, le juge ne peut statuer qu'après avoir entendu ou appelé le curateur » (NCPC. art. 1263)

Mais d'autres fois la protase suit l'apodose :

« La vente de la chose d'autrui est nulle [...] lorsque l'acheteur a ignoré que la chose fût à autrui » (C. civ. art. 1599) 
Parfois la condition est négative :

«Il peut être renouvelé, sauf dans le cas visé par le deuxième alinéa de l'article 173 ci-dessus » (C. civ. art. 176)

\section{La substantivation et la dérivation dans le vocabulaire juridique}

C'est la propension à la nominalisation à cause du besoin d'identifier les parties d'un procès ou les sujets intervenant dans des actes juridiques. Gérard Cornu a lui-même expliqué ce fait dans son Vocabulaire juridique (1990). Dans les cas où la langue générale n'a pas de terme approprié, le langage juridique se sert du néologisme et de la polysémie utilisant notamment des substantifs dérivés d'adjectifs ou participes (substantivation) ou en ajoutant des préfixes ou suffixes aux substantifs existants (dérivation).

«L'une des caractéristiques de la terminologie juridique par rapport aux autres terminologies spécialisées est en effet l'importance de la polysémie. Le caractère souverain des textes législatifs leur donnant le pouvoir de nommer, le législateur crée donc les termes qui correspondent aux notions juridiques nouvelles » (Dinh Phuc \& Meunier-Crespo : 2008)

Au procès civil, celui qui introduit une demande en justice porte le nom de demandeur, demanderesse au féminin, et la personne contre la quelle est intentée l'action est le défendeur (défenderesse au féminin), mais celui qui prend l'initiative devant la Cour d'Appel porte le nom d'appelant, tandis que le défendeur à l'instance d'appel est l'intimé.

\section{La substantivation}

Consiste à transformer en nom un verbe ou un adjectif pour identifier les parties engagées dans un procès :

« Pour une large part, le droit règle l'office ou le sort de ses protagonistes en formalisant le rôle des acteurs (le créancier, le débiteur) comme le théâtre classique formalise celui de ses personnages, jusque dans ses «utilités» (le confident, la servante, l'amant, etc.) » (Cornu, $1990: 158)$.

Substantivation du participe présent : le participe présent indique l'action.

«Le tribunal peut, toutefois, à la demande de l'adoptant, décider que l'adopté ne portera que le nom de l'adoptant » (Code civil, Art. 363)

\begin{tabular}{|l|l|l|}
\hline Verbe & Action (substantif) & Sujet (substantif) \\
\hline accepter & Acceptation & acceptant \\
\hline appeler & Appel & appelant \\
\hline attaquer & Attaque & attaquant \\
\hline gérer & Gestion & gérant \\
\hline payer & Payement & payant \\
\hline
\end{tabular}

Substantivation du participe passé : le participe passé indique la réception d'un bénéfice ou la position réceptive.

«La commune est responsable du dommage subi par le prévenu » (Tribunal d'instance, Aix-en-Provence, $11 / 04 / 1989)$

\begin{tabular}{|l|l|l|}
\hline Adopté & Obligé & Prévenu \\
\hline Fiancé & Saisi & Inculpé \\
\hline
\end{tabular}




\begin{tabular}{|l|l|l|}
\hline Marié & Engagé & Condamné \\
\hline Associé & Intimé & Subrogé \\
\hline
\end{tabular}

Dans certains cas, il y a une correspondance entre le participe présent et le participe passé (adoptant / adopté), mais cela ne constitue une règle générale :

\begin{tabular}{|l|l|}
\hline \multicolumn{1}{|c|}{ La position active } & La réception d'un profit \\
\hline adjudicateur & adjudicataire \\
\hline appelant & intimé \\
\hline bailleur & preneur \\
\hline cédant & cessionnaire \\
\hline créancier & débiteur \\
\hline demandeur & défendeur \\
\hline donateur & donataire \\
\hline mandant & mandataire \\
\hline prêteur & emprunteur \\
\hline testateur & héritier / légataire \\
\hline usufruitier & nu-propriétaire \\
\hline vendeur & acquéreur \\
\hline
\end{tabular}

\section{La dérivation}

La dérivation consiste à former un mot nouveau depuis un mot de base, soit par addition, soit par suppression, soit par remplacement d'éléments appelés préfixes et suffixes. Les préfixes et les suffixes ajoutés au radical sont des éléments largement utilisés dans le langage juridique.

Le suffixe -eur/-eresse/-euse/-ice marque l'action, l'initiative, la position active :

«L'agent immobilier mandataire de la bailleresse [...] transgresse son obligation de renseignement en n'informant pas la bailleresse des conséquences d'un congé donné pour un faux motif » (Cour d'appel, Paris, Chambre 6, section B, 28/09/1990)

\begin{tabular}{|l|l|l|}
\hline Bail & bailleur & bailleresse \\
\hline Débit & débiteur & débitrice \\
\hline Demande & demandeur & demanderesse \\
\hline Donation & donateur & donatrice \\
\hline Emprunt & emprunteur & emprunteuse \\
\hline Louage & loueur & loueuse \\
\hline Plaidoirie & plaideur & plaideuse \\
\hline Prêt & prêteur & prêteuse \\
\hline Sollicitation & solliciteur & solliciteuse \\
\hline Testament & testateur & testatrice \\
\hline
\end{tabular}

Le suffixe-aire signale la réception d'un profit ou le titulaire d'un droit : 
«Les donataires, les légataires, ni même les héritiers légitimes de celui qui aura fait la disposition, ni pareillement leurs donataires, légataires ou héritiers, ne pourront, en aucun cas, opposer aux appelés le défaut de publication ou inscription » (code civil 1072)

«La requête est remise ou adressée au secrétariat-greffe par le requérant ou par tout mandataire » (code de procédure civile 852)

\begin{tabular}{|l|l|}
\hline Allocation & Allocataire \\
\hline Bénéfice & Bénéficiaire \\
\hline Cession & Cessionnaire \\
\hline Dépôt & Dépositaire \\
\hline Donation & Donataire \\
\hline Mandat & Mandataire \\
\hline Réserve & Réservataire \\
\hline
\end{tabular}

\section{Les définitions}

En Droit anglo-saxon, il y a une tradition de définir les termes utilisés dans chaque texte législatif, tradition qu'on utilise aussi en droit international, notamment aux traités internationaux.

En Droit français, on trouve une grande quantité de définitions : il y a plus de cent définitions au Code civil et aussi dans d'autres codes, lois, décrets, etc.

Dans la plupart des cas, la définition fait référence aux termes juridiques (mineur, société, usufruit, etc.), mais aussi aux collocations (condition résolutoire, contrat aléatoire, etc.).

Définition de mineur (Art. 388 C. c.) :

«Le mineur est l'individu de l'un ou de l'autre sexe qui n'a point encore l'âge de dix-huit ans accomplis ».

Définition de société (Art. 1832 C.c.) :

«La société est instituée par deux ou plusieurs personnes qui conviennent par un contrat d'affecter à une entreprise commune des biens ou leur industrie en vue de partager le bénéfice ou de profiter de l'économie qui pourra en résulter ».

Définition d'usufruit (Art. 578 C.c.) :

«L'usufruit est le droit de jouir des choses dont un autre a la propriété, comme le propriétaire lui-même, mais à la charge d'en conserver la substance ».

Définition de servitude (Art. 637 C.c.) :

«Une servitude est une charge imposée sur un héritage pour l'usage et l'utilité d'un héritage appartenant à un autre propriétaire ».

Définition de condition résolutoire (Art. 1183 C.c.) :

« La condition résolutoire est celle qui, lorsqu'elle s'accomplit, opère la révocation de l'obligation, et qui remet les choses au même état que si l'obligation n'avait pas existé ».

Définition de contrat aléatoire (Art. 1964 C.c.) :

«Le contrat aléatoire est une convention réciproque dont les effets, quant aux avantages et aux pertes, soit pour toutes les parties, soit pour l'une ou plusieurs d'entre elles, dépendent d'un événement incertain ». 


\section{Références bibliographiques}

Austin, J.L. (1970). Quand dire c'est faire. Paris : Seuil. 1962. Trad. fr. 1970, rééd. Seuil, coll. « Points essais », 1991.

Champeil-Desplats, V. (2006). La normativité. N'est pas normatif qui peut. L'exigence de normativité dans la jurisprudence du Conseil constitutionnel, Cahiers du Conseil constitutionnel, 21, Etudes et doctrines.

Charrow, V.R., Crandall, J.A. \& Charrow, R.P. (1982). Characteristics and Functions of Legal Language. In Sublanguage: Studies of Language in Restricted Semantic Domains, éd. R. Kittredge \& J. Lehrberger, 175-190. Berlin : W. de Gruyter.

Cornu, G. (1990). Vocabulaire juridique. Paris : Dalloz.

Desmet, I. (2006). Variabilité et variation en terminologie et langues spécialisées : discours, textes et contextes. Mots, termes et contextes. Actes des septièmes Journées scientifiques du réseau de chercheurs Lexicologie, Terminologie, Traduction, coll. « Actualité scientifique », D. Blampais, Philippe Thoiron, Marc van Campenhoudt (éds.). Paris : Éditions des archives contemporaines et Agence universitaire de la Francophonie, 235-247.

Dinh Phuc, L. \& Meunier-Crespo, M. (2008). Présentation d'une recherche linguistique sur le Code des délits et des peines (1810) et sur le Code pénal (1994). Les mercredis du CEL, Lyon.

Garapon, A. (1997). Bien juger, essai sur le rituel judiciaire. Paris : éd. Odile Jacob.

Gémar, J.C. (1991). Terminologie, langue et discours juridiques. Sens et signification du langage du droit. Meta, XXXVI-I pp. 275-283

Greimas, A. J. (1966). Eléments pour une théorie de l'interprétation du récit mythique. In Communications, 8: L'Analyse structurale du récit. Paris : Seuil, 1981, pp. 34-65.

Houbert, F. (2004). Le triple défi du traducteur juridique. American Translators Association, Revue de la common law en français, 5/2, 607-618.

Kalinowski, G. (1972). La logique des normes. Paris : PUF.

Malkiel, Y. (1959). Studies in Irreversible Binomials. Lingua 8, 113-160.

Martinet, A. (1969). La Linguistique. Guide Alphabétique. Avec la collaboration de Jeanne Martinet et Henriette Walter. [Collection Guides Alphabétiques Médiations] Paris: Éditions Denoël.

Sourioux, J.-L. \& Lerat, P. (1975). Le langage du droit. Paris : PUF.

Terré, F. (1991). Introduction générale au droit. Précis Dalloz, ${ }^{\circ} 150$.

Thibierge, C. (2003). Le droit souple. RTD Civ. Dalloz.

\footnotetext{
${ }^{1}$ M. Jean Foyer : « [...] Cette semaine, le ministre d'Etat, ministre de la recherche et de la technologie, nous présente un projet dont je dirai, ne parlant pas latin pour une fois (sourires), mais empruntant ma terminologie à la langue des physiciens, qu'il est pour l'essentiel un assemblage de neutrons législatifs, je veux dire de textes dont la charge juridique est nulle » (Assemblée Nationale, 3e séance du 21 juin 1982, compte rendu intégral, orientation de la recherche et du développement technologique. Suite de la discussion d'un projet de loi adopté par le Sénat après déclaration d'urgence. p. 3667).

${ }^{2}$ Décision $\mathrm{n}^{\circ}$ 2005-512 DC du 21 avril 2005. Annexe $1:$ absence de normativité ou normativité incertaine de la loi.

${ }^{3}$ Décision $n^{\circ}$ 2005-512 DC du 21 avril 2005. Annexe 1 : absence de normativité ou normativité incertaine de la loi.

4 « Au point de vue matériel, la loi se définit comme une règle abstraite : elle est formulée au sujet de situations typiques énoncées in abstracto. Ce caractère est lié à la généralité de la loi : la règle promulguée a un caractère général en ce qu'elle est destinée non un cas particulier mais une série de cas semblables susceptibles de se présenter » (Terré : 1991).
} 